\title{
CHILD HOOD OBESITY - A LIFELONG THREAT TO HEALTH.
}

\author{
${ }^{*}$ Mrs.Geetha.C.** Dr. S.Jeyalakshmi.*** Dr.S.Kamalam.
}

\section{Abstract:}

Recently, high prevalence of overweight and obesity has been reported in children and adolescents both in developed and in developing countries and these figures have been projected to increase further in coming years. Childhood obesity increases the risk of adulthood obesity and obesity related diseases such as hypertension, diabetes mellitus, and dyslipidemia. Several studies highlight recent evidence regarding the role of genetics, age, gender, birth weight, dietary pattern: eating fast foods and snacks, skipping breakfast and behavioral characteristics: sedentary activities, physical activity pattern, and sleeping hours related to occurrence of obesity in children and adolescents. Interventions related to changing dietary practices and maintenance of regular physical activity among children through parental initiative and social support in natural settings such as at home and in schools, or afterschool care services are the most important to prevent childhood obesity.

Keywords: Obesity; Children; Determinants; Prevention; Genetics; Physical activity; Dietary pattern.

\section{INTRODUCTION}

Childhood obesity is a serious medical condition that affects children and adolescents. It occurs when a child is well above the normal weight for his/her age and height. Obesity in childhood and adolescents has adverse consequence on premature mortality and physical morbidity in adulthood and is associated with impaired health during childhood.8 The important obesity risk factors in which different studies revealed genetic history, physical activity, high birth weight, and type of milk of consumed during infancy, more than two hours television watching per day, number of regular meals and parental overweight as the risk factors ofobesity. 3

\section{Prevalence of childhood obesity}

Childhood obesity is one of the most serious public health challenges of the 21 st century. The prevalence of obesity is increasing both in developed and in developing countries and has become a major public health problem. Up to 1980s in developing countries were with the lowest rates, but since then overweight and obesity prevalence have gradually increased in children. The global prevalence of overweight and obesity in children aged 5 17 years is $10 \%$ and this global average covers a wide range of prevalence levels in different regions and countries with above $30 \%$ in America and below 2\% in Sub Saharan Africa Further, projections to the year 2010 for estimated prevalence of Overweight and obesity in school age children (aged 5 -17 years) are with 46\% in America and below 5\% in Africa .For children, between 5-17 years in this regional prevalence data onoverweight 
and obesity are currently unavailable However, data for overweight and obesity prevalence among children in different countries in South Asia are available: $25.0 \%$ among children from 2 to 15 years in Bangladesh and $22.0 \%$ among children from 5 to 19 years in India. Moreover, secular trends indicate increasing prevalence rates in these countries: for example, 9.8 to $11.7 \%$ among children from 5 to 19 years in India during2006-2009

\section{Physiology of obesity}

The normal growth of adipose tissue takes account of several phases. During first year (early infancy) of the first period, adiposity increases and it reduces in next year and remains stable for several years. Adiposity rebound start at around 6 years during second period. From this stage, both size and number of adiposities increases .Therefore, childhood is the critical period to develop obesity because of the adiposity rebound.

Obesity negatively influences a child's self-esteem and results in diminished quality of life. Moreover, children with high body mass index (BMI) often become obese adults, who are at increased risk of developing obesity-related diseases, such as type 2 diabetes, hypertension, dyslipidemia and certain types of cancer and place significant financial burden on healthcare systems. For measuring healthy body weight, it is difficult to develop one simple index for overweight and obesity in children and adolescents because their bodies undergo a number of physiological changes as theygrow.

\section{Different methods to measure a body's healthyweight \\ Anthropometric measures of relative fatness}

Among the anthropometric measures of relative adiposity or fatness are waist, hip and other girth measurements, skin fold thickness and indices derived from measured height and weight such as Quetelet's index (BMI or W $\mathrm{H}-2$ ), the ponderal index (W $\mathrm{H}-3$ ) and similar formulae.

\section{Weight forheight}

Low weight for height is termed thinness, and very low weight for height is termed wasting, usually found as a consequence of acute starvation and/or disease .A high weight for height is termed overweight and very high weight for height is termed obese.

\section{Weight and weight-for-height}

Total body weight can be recorded and compared with reference standards based on a child's age.. Weight-for-height charts are inaccurate beyond the age of around 10-11 years and the measure is not useful in older children and adolescents. Weight and height (or length) are relatively easy to obtain, although they tend to be more accurate if taken by a trained person. Weight should be taken with the child in light, indoor clothing.

\section{BMI}

Body mass index (BMI) is defined as weight (kg) /height squared (m2), and is widely used as an index of relative adiposity among children, adolescents and adults. Among adults, the WHO recommends that a person with a BMI of $25 \mathrm{~kg} \mathrm{~m}-2$ or above is classified overweight, while one with a BMI $30 \mathrm{~kg} \mathrm{~m}-2$ or above is classified obese although revisions of these guidelines are being proposed for certain populations .

\section{BMI for age reference charts and BMI for agepercentiles.}

The advantage of using BMI-for-age charts is that a child can be described as being above or below certain percentile lines (for example the 85th or $90^{\text {th }}$ percentile), which can be useful in a clinical setting. Children with a BMI greater than or 
equal to the $95^{\text {th }}$ percentile be classified as 'overweight' and those children with a BMI between the 85th and 95th percentile be classified as 'at risk of overweight. Body mass index should be used as the main measure of overweight and obesity in childhood and adolescence for survey purposes.

\section{World Health Organization (WHO) definitions for obesity}

$B M I=$ Weight $\mathrm{kg} /$ Height $\mathrm{m}^{2}$

- Underweight: $<18.5 B M I$

- Healthy weight: 18.5-24.9BMI

- Overweight (Grade I obesity) : 25.0-29.9BMI

- Obese (Grade II): 30.0-39.9BMI

- Morbidly obese (GradeIII): 40 or aboveBMI

- $\quad$ Super obese (Grade IV): BMI>50

BMI is significantly associated with relative fatness in childhood and adolescence, and is the most convenient way of measuring relativeadiposity

\section{WAIST-TO HIPRATIO}

Waist circumference and Waist-to hip ratio (WHR) Waist circumference is an indirect measure of central adiposity. Central adiposity is strongly correlated with risk for cardiovascular disease in adults and an adverse lipid profile and hyper insulinaemia in children. Waist circumference is measured at the minimum circumference between the iliac crest and the rib cage using an anthropometric tape-to-hip ratio has been used among adults to identify people with high central adiposity. Waist circumference is measured as above and hip circumference is measured at the maximum protuberance of the buttocks. The ratio is thencalculated.

\section{SKIN-FOLDTHICKNESS}

Skin-fold thickness can be measured at different sites on the body (triceps, sub scapular) using skin-fold callipers.

\section{Determinants of Childhood Overweight / Obesity}

Many studies indicate several key determinants widely acknowledged as the main drivers of the obesity epidemic among children.

\section{Genetics}

Obesity tracks in families, and one of the strongest predictors of child overweight is the BMI of the mother and father. In recent years, progress was made in identifying genes that may contribute to this effect. A recent study showed for association with the FTO (fat mass and obesity-associated) gene and found strong associations with BMI and weight among children. Moreover, a defect in the melanocortin4 receptor gene (MC4R) is associated with a severe and early form of monogenic obesity inchildren

\section{Age and gender}

Age and gender have been identified as key determinants for the development of obesity. A study conducted among 2-18 children in Nigeria revealed that Males had higher BMI than females at age group 2-6 years, whereas females had higher BMI than males at age groups 11-14 years and 15-18 years.

\section{Birth weight}

Rapid weight gain which was traditionally considered as a healthy intervention for low birth weight infants is now recognized as a potential risk factor of increasing interest for obesity; In the geographically defined birth cohort of the Avon longitudinal study of pregnancy and childhood (ALSPAC), it showed that early postnatal catch-up growth, between birth and two years, is a risk factor for childhood obesity and may therefore contribute to the greatest risk for disease in adulthood

\section{DIETARY PATTERN}

\section{Eating fast foods and snacks:}

Fast foods play as a key contributor to the rising prevalence of obesity among children 
because of fast food's poor nutritional quality, as fast foods have higher total energy, total fat, and saturated fat intakes; have refined carbohydrates and lower fibre intakes; and with higher energy density . Further, fast food consumption is also associated with higher intake of sugars sweetened beverages and French fries and lower intake of milk, fruit , and vegetables.

\section{Skipping breakfast:}

Breakfast is a most important meal to start a day. Skipping breakfast leads to hunger and increases the amount of lunch. Therefore, it leads to play a key role in causing obesity in children Some studies showed the higher prevalence among students who skip breakfast than others . Ortega et al., identified through 7 day food records that obese subject omit breakfast than normal person. It suggests that inadequate food choices contribute to poor food choices for rest of the day and it increases the risk of obesity in longterm.

\section{Behavioural Characteristics}

\section{Sedentary activities and screen viewing}

Rapid increase in childhood obesity has also been attributed to a shift in the activity patterns from outdoor play to indoor entertainment: television viewing, internet, and computer games. A study suggested that decreasing any type of sedentary time is associated with lower health risk in youth aged 5-17 years. In particular, the evidence suggested that daily TV viewing in excessof 2 hours is associated upward in BMI. Another study showed that Overweight and obese children were more sedentary and higher screen time than normal weight children . Further, a European youth heart study conducted among 9-10 year old boys and girls found the significant positive relationships between $\mathrm{TV}$ viewing and adiposity after adjusting for gender, age group, study location, sexual maturity and birth weight . Mitchell et al. studied the association of hours of objectively measured sedentary behavior and odds of being obese and confirmed that sedentary behaviour was positively associated with obesity.

\section{Physical activity pattern}

Physical activity plays an important role in protection from obesity .A study revealed that Prevalence of overweight and obese was higher among children who travel to school in motor vehicle than children who travel by cycle or walk. Another study done in Iran reported significant difference in physical activity among obese and nonobese children. Non-obese children engaged with more physical activities like running, football, and travel to school by foot than obese children did.

\section{Sleeping hours}

In school-age children, several studies have consistently reported that short sleep duration was an independent risk factor for obesity A cross-sectional study conducted among 229 Mexican American 8-10-yearolds and concluded that children who slept less were more likely to have a higher BMI Z-Score . A cohort study done in Japan observed positive relationship between decreased sleeping hours and obesity after adjusting for potential confounding factors .

\section{Adverse Health Outcomes in Children}

As the prevalence and severity of childhood obesity increase, concern about adverse health outcomes in childhood and adolescence is rising. obesity is linked with many disorders of the metabolic system. Such disorders, heretofore seen primarily in adulthood, are now appearing in children. Even when the disorders do not present themselves in childhood, childhood obesity or overweight increases the risk of their developing in adulthood. Much the same generalization applies to the obesity-related disorders in the other bodily systems. 
A well-established pulmonary consequence of childhood obesity is 'sleep-associated breathing disorder', most clearly seen in severe obesity

\section{Physical consequences of childhood and adolescent obesity}

\section{PULMONARY}

- Sleepapnoea

- Asthma

- Pickwickiansyndrome

- Increased bronchialhyperactivity

- Asthmaexacerbation

- Pulmonaryembolism

- Increased sensitivity tosodium

- Decreasednatriuresis

- Proteinuria

- Focal segmental glomerulosclerosis(FSGS)

\section{ORTHOPAEDIC}

- Slipped capitalepiphyses

- Blount's disease (tibiavara)

- Tibialtorsion

- Flat feet, Anklesprains

- Increased risk offractures

- Osteoarthritis

\section{NEUROLOGICAL}

- Idiopathic intracranial hypertension

(e.g. pseudotumourcerebri)

\section{GASTROENTEROLOGICAL}

- Cholelithiasis

- Liver steatosis / non-alcoholic fattyliver

- Gastro-oesophageal refluxGallstones

- Non alcoholicsteato hepatitis(NASH)

- Hepatic fibrosis

- Cirrhosis

\section{ENDOCRINE}

- Insulin resistance/impaired glucosetolerance

- Type 2diabetes

- Menstrualabnormalities

- Polycystic ovarysyndrome

- Hypercorticism
- Insulinresistance

- Diabetes mellitus(NIDDM)

\section{CARDIOVASCULAR}

- Hypertension

- Dyslipidaemia

- Fattystreaks

- Left ventricularhypertrophy

- High bloodpressure

- Early onset ofatherosclerosis

PSYCHOSOCIAL

- Obsessive concern about bodyimage

- Expectation ofrejection

- Progressivewithdrawal

- Low self esteem.,Depression

\section{STRATEGIES FOR CHILDHOOD OBESITY PREVENTION}

Obesity prevention is not simply an issue of individual responsibility and prevention strategies seem to be more effective in children than in adults. Therefore, Successful strategies for obesity prevention among children should be targeted and implemented in natural settings for influencing the diet and physical activities at home, in preschool institutions, schools, or after-school care services.

\section{School-Based Strategies}

School-based programs have great opportunity to promote healthy nutrition and physical activity because most children attend school and a child spends more than half of his/her waking hours at school on any given school day. Therefore, following school-based interventions should be implemented as standard practice in educational settings improvements in knowledge, attitudes, and behaviour:

- Components on healthy eating, physical activity and body image should be integrated into the regularcurriculum. 
- Sessions for physical activity and the development of fundamental movement skills should be included throughout the schoolweek.

- The nutritional quality of foods made available to students (e.g.in school canteens) should be closely monitored and improved.

- An environment and culture should be created that supports children eating nutritious foods and being active throughout eachday.

- Parents should be engaged to support activities in the home setting to encourage children to become more active, eat more nutritious foods and spend less time in screen-basedactivities.

\section{Home-Based Strategies}

Parental initiative is necessary to succeed the home-based strategies influencing the diet and physical activity among children. Therefore, parents should be aware about following activities:

- Should be good rolemodels.

- Should encourage the children to have regular meals including breakfast at home, because children who take part in family meals are also more likely to eat fruits, vegetables, and grains and less likely to snack on unhealthyfoods.

- Should provide the children with healthy foodchoices.

- Should encourage young children to develop good eating habits and preferences for healthy foods because eating behaviours that develop during childhood tend to track intoadulthood.

- Should accompany children to nearby parks and play with them. This would not only add to their health benefits but also let them supervise their children. Further, it helps to limit sedentaryactivities.

- Should involve the children in food purchasing by taking children to food shopping and allowing them to select healthyfoods

\section{CONCLUSION}

Childhood obesity leads to its related noncommunicable diseases and psychosocial health problems in children. This is because Childhood obesity is a crucial issue that needs to be addressed urgently. Changing dietary practices and maintenance of regular physical activity starting as early as infancy through parental initiative and social support interventions are the most important strategies to tackle childhood obesity.

\section{REFERENCES}

1. Lasserre M, Arnald C, Pacaud P, Pascal B (2007) Worldwide trends in childhood obesity. Swiss Med Wkl 137:157-158.

2. Wang Y, Lobstein T (2006) Worldwide trends in childhood overweight and obesity. International Journal of Pediatric Obesity 1:11-25.

3. deWlide JA, Zandbergen-Harlaar S, van Buuren S, Middlekoop BJ (2013) Trends in body mass index distribution and prevalence of thinness, overweight

and obesity in two cohorts of Surinamese South Asian children in The Netherlands. Arch Dis Child 98: 280-285.

4. Bulbul T, Houque M (2014) Prevalence of childhood obesity \& overweight in Bangladesh findings from a countrywide epidemiological study. BMC Pediatrics 14:86.

5. Guptha N, Goel K, Shah P, Mishra A (2012) Childhood Obesity in Developing Countries: Epidemiology, Determinants, and Prevention. Endocrine Reviews 33:48-70.

6. Thilakarathne RMLR, Wijesinghe DGNG (2011) Association between Nutritional Status and Life Style Practices of Primary School Children in the Colombo District: A Pilot Study. Tropical Agricultural Research 22:392-401.

7. Wickramasinghe VP, Lamabadusuriya SP, Atapattu N, Sathyadas G, Karuparanantha S, et al. (2004) Nutritional status of school children in an urban area of Sri Lanka. Ceylon Medical Journal49: 